\title{
Verification of Cryptographic Protocols: Tagging Enforces Termination
}

\author{
Bruno Blanchet ${ }^{1,2}$ and Andreas Podelski ${ }^{2}$ \\ 1 Département d'Informatique \\ École Normale Supérieure, Paris \\ Bruno.Blanchet@ens.fr \\ 2 Max-Planck-Institut für Informatik, Saarbrücken \\ podelski@mpi-sb.mpg.de
}

\begin{abstract}
In experiments with a resolution-based verification method for cryptographic protocols, we could enforce its termination by tagging, a syntactic transformation of messages that leaves attack-free executions invariant. In this paper, we generalize the experimental evidence: we prove that the verification method always terminates for tagged protocols.
\end{abstract}

\section{Introduction}

The verification of cryptographic protocols is an active research area, see [122 . It is important since the design of protocols is error-prone, and testing cannot reveal potential attacks against the protocols. In this paper, we study a verification technique based on Horn clauses and resolution akin to [4/524]. We consider a protocol that is executed in the presence of an attacker that can listen to the network, compute, and send messages. The protocol and the attacker are translated into a set of Horn clauses such that: if the fact $\operatorname{att}(M)$ is not derivable from the clauses, then the protocol preserves the secrecy of the message $M$ in every possible execution. The correctness verified is stronger than the one required since the executions possible in the Horn clause model include the ones where a send or receive instruction can be applied more than once in the same session. In practice, the difference between the correctness criteria does not show (no false alarm arised in our experiments).

The verification technique consists of the translation into Horn clauses, followed by the checking of the derivability of facts att $(M)$ by a resolution-based algorithm. It has the following characteristics.

- It can verify protocols with an unbounded number of sessions.

- It can easily handle a variety of cryptographic primitives, including sharedkey and public-key cryptography (encryption and signatures), hash functions, message authentication codes (mac), and even a simple model of DiffieHellman key agreements. It can also be used to verify authenticity [5].

- It is efficient (many examples of protocols of the literature are verified in less than $0.1 \mathrm{~s}$; see [5]). 
The resolution-based verification algorithm has one drawback: it does not terminate in general. In fact, in our experiments, we detected infinite loops during its application to the Needham-Schroeder shared-key protocol [4] and several versions of the Woo-Lam shared-key one-way authentication protocol [5]. It is always possible to modify the algorithm to make it work on those cases and any finite number of other cases, but that will not affect its inherent non-termination property (inherent by the undecidability of the problem that it tries to solve). In this paper, we investigate an alternative: tagging the protocol.

Tagging consists in adding a unique constant to each message. For instance, to encrypt the message $m$ under the key $k$, we add the tag $\mathrm{c}_{0}$ to $m$, so that the encryption becomes sencrypt $\left(\left(c_{0}, m\right), k\right)$. The tagged protocol retains the intended behaviour of the original protocol; i.e., the attack-free executions are the same. Under attacks, it is possibly more secure. Therefore, tagging is a feature of a good protocol design, as explained e.g. in [2]: the receiver of a message uses the tag to identify it unambiguously; thus tagging prevents type flaws that occur when a message is taken for another message. (This is formally proved in [16] for a tagging scheme very similar to ours.) Tagging is also motivated by practical issues: the decoding of incoming messages becomes easier. For all these reasons, tags are already present in protocols such as SSH.

In our experiments (including the protocols mentioned above), we obtained termination after tagging the protocol. In this paper, we give the theory behind the experiments: the resolution-based verification algorithm always terminates on tagged protocols. More precisely, on protocols where tags are added to each use of a cryptographic primitive, which may be among: public-key cryptography where keys are atomic, shared-key cryptography (unrestricted), hash functions, and message authentication codes (mac's).

This means that we show termination for a class of protocols that includes many relevant examples.

\section{Horn Clauses Representing a Protocol}

This section and the next one recapitulate the necessary background on the translation and the algorithm, using material from [4].

Cryptographic protocols can be translated into Horn clauses, either by hand, as explained in 424], or automatically, for instance, from a representation of the protocol in an extension of the pi calculus, as in [1].

The terms in the Horn clauses stand for messages. The translation uses one predicate att. The fact att $(M)$ means that the attacker may have the term $M$. The fundamental property of this representation is that if $\operatorname{att}(M)$ is not derivable from the clauses, then the protocol preserves the secrecy of $M$.

The clauses are of two kinds: the clauses in $\mathcal{R}_{\text {Primitives }}$ that depend only on the signature of the cryptographic primitives (they represent computation abilities of the attacker) and the clauses in $\mathcal{R}_{\text {Prot }}$ that one extracts from the protocol itself. 


\section{Tuples:}

Constructor: tuple $\left(M_{1}, \ldots, M_{n}\right)$

Destructors: projections $i \operatorname{th}_{n}\left(\left(M_{1}, \ldots, M_{n}\right)\right) \rightarrow M_{i}$

Shared-key encryption:

Constructor: encryption of $M$ under the key $N$, sencrypt $(M, N)$

Destructor: decryption sdecrypt $(\operatorname{sencrypt}(M, N), N) \rightarrow M$

Public-key encryption:

Constructors: encryption of $M$ under the public key $N$, pencrypt $(M, N)$

public key generation from a secret key $N, \operatorname{pk}(N)$

Destructor: decryption pdecrypt(pencrypt $(M, \operatorname{pk}(N)), N) \rightarrow M$

Signatures:

Constructor: signature of $M$ with the secret key $N, \operatorname{sign}(M, N)$

Destructors: signature verification checksignature $(\operatorname{sign}(M, N), \operatorname{pk}(N)) \rightarrow M$ message without signature getmessage $(\operatorname{sign}(M, N)) \rightarrow M$

Non-message-revealing signatures:

Constructors: signature of $M$ with the secret key $N, \operatorname{nmrsign}(M, N)$ constant true

Destructor: signature verification $\operatorname{nmrchecksign}(\operatorname{nmrsign}(M, N), \operatorname{pk}(N), M) \rightarrow \operatorname{true}$

One-way hash functions:

Constructor: hash function hash $(M)$.

Message authentication codes, keyed hash functions:

Constructor: mac of $M$ with key $N, \operatorname{mac}(M, N)$

Fig. 1. Constructors and destructors

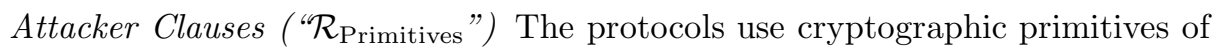
two kinds: constructors and destructors (see Figure 1). A constructor $f$ is used to build up a new term $f\left(M_{1}, \ldots, M_{n}\right)$. For example, the term sencrypt $(M, N)$ is the encoding of the term $M$ with the key $N$ (by shared-key encryption). A destructor $g$ applied to terms $M_{1}, \ldots, M_{n}$ yields a term $M$ built up from subterms of $M_{1}, \ldots, M_{n}$. It is defined by a finite set $\operatorname{def}(g)$ of equations written as reduction rules $g\left(M_{1}, \ldots, M_{n}\right) \rightarrow M$ where the terms $M_{1}, \ldots, M_{n}, M$ contain only constructors and variables. For example, the rule $\operatorname{sdecrypt}(\operatorname{sencrypt}(M, N), N) \rightarrow$ $M$ models the decoding of the term sencrypt $(M, N)$ with the same key used for the encoding.

The attacker can form new messages by applying constructors and destructors to already obtained messages. This is modeled, for instance, by the following clauses for shared-key encryption.

$$
\begin{aligned}
& \operatorname{att}(x) \wedge \operatorname{att}(y) \rightarrow \operatorname{att}(\operatorname{sencrypt}(x, y)) \\
& \operatorname{att}(\operatorname{sencrypt}(x, y)) \wedge \operatorname{att}(y) \rightarrow \operatorname{att}(x)
\end{aligned}
$$

The first clause expresses that if the attacker has the message $x$ and the shared key $y$, then he can form the message sencrypt $(x, y)$. The second clause means that if the attacker has the message sencrypt $(x, y)$ and the key $y$, then he can obtain the message $x$ (by applying the destructor sdecrypt and then using the equality between sdecrypt(sencrypt $(x, y), y)$ and $x$ according to the reduction rule for sdecrypt). 
We furthermore distinguish between data and cryptographic constructors and destructors and thus, in total, between four kinds of primitives. The set DataConstr of data constructors contains those $f$ that come with a destructor $g_{i}$ defined by $g_{i}\left(f\left(x_{1}, \ldots, x_{n}\right)\right) \rightarrow x_{i}$ for each $i=1, \ldots, n$; i.e. $g_{i}$ is used for selecting the argument of $f$ in the $i$-th position. It is generally sufficient to have only tuples as data constructors (with projections as destructors). All other constructors are said to be cryptographic constructors; they form the set CryptoConstr. We collect all clauses like the two example clauses above, for each of the four cases, in the set $\mathcal{R}_{\text {Primitives }}$ of clauses or rules defined below.

Definition 1 (Program for Primitives, $\mathcal{R}_{\text {Primitives }}$ ). The program for primitives, $\mathcal{R}_{\text {Primitives, }}$ is the union of the four sets of Horn clauses corresponding to each of the four cases of cryptographic primitives:

$-\mathcal{R}_{\text {CryptoConstr }}$ is the set of clauses $\operatorname{att}\left(x_{1}\right) \wedge \ldots \wedge \operatorname{att}\left(x_{n}\right) \rightarrow \operatorname{att}\left(f\left(x_{1}, \ldots, x_{n}\right)\right)$ where $f$ is a cryptographic constructor.

$-\mathcal{R}_{\text {DataConstr }}$ is the set of clauses $\operatorname{att}\left(x_{1}\right) \wedge \ldots \wedge \operatorname{att}\left(x_{n}\right) \rightarrow \operatorname{att}\left(f\left(x_{1}, \ldots, x_{n}\right)\right)$ where $f$ is a data constructor.

$-\mathcal{R}_{\text {CryptoDestr }}$ is the set of clauses att $\left(M_{1}\right) \wedge \ldots \wedge$ att $\left(M_{n}\right) \rightarrow \operatorname{att}(M)$ where $g$ is a cryptographic destructor with the reduction rule $g\left(M_{1}, \ldots, M_{n}\right) \rightarrow M$.

$-\mathcal{R}_{\text {DataDestr }}$ is the set of clauses $\operatorname{att}\left(f\left(x_{1}, \ldots, x_{n}\right)\right) \rightarrow \operatorname{att}\left(x_{i}\right)$ where $f$ is a data constructor and $i=1, \ldots, n$.

Protocol Clauses ("R $\mathcal{R}_{\text {Prot }}$ ) We note $\mathcal{R}_{\text {Prot }}$ the set of protocol clauses. These include clauses that directly correspond to send and receive instructions of the protocol and clauses translating the initial knowledge of the attacker.

In a protocol clause of the form

$$
\operatorname{att}\left(M_{1}\right) \wedge \ldots \wedge \operatorname{att}\left(M_{n}\right) \rightarrow \operatorname{att}(M)
$$

the term $M$ in the conclusion represents the sent message. The hypotheses correspond to messages received by the same host before sending $M$. Indeed, the clause means that if the attacker has $M_{1}, \ldots, M_{n}$, he can send these messages to a participant who is then going to reply with $M$, and the attacker can then intercept this message.

If the initial knowledge of the attacker consists of the set of terms $S_{\text {Init }}$ (containing e.g. public keys, host names, and a name $\mathrm{N}$ that represents all names that the attacker creates), then it is represented by the facts att $(M)$ where $M$ is a term in $S_{\text {Init }}$.

We explain protocol clauses on the example of the Yahalom protocol [8]:

$$
\begin{array}{lll}
\text { Message 1. } & A \rightarrow B: & \left(A, N_{a}\right) \\
\text { Message 2. } & B \rightarrow S: & \left(B,\left\{A, N_{a}, N_{b}\right\}_{K_{b s}}\right) \\
\text { Message 3. } & S \rightarrow A: & \left(\left\{B, K_{a b}, N_{a}, N_{b}\right\}_{K_{a s}},\left\{A, K_{a b}\right\}_{K_{b s}}\right) \\
\text { Message 4. } & A \rightarrow B: & \left(\left\{A, K_{a b}\right\}_{K_{b s}},\left\{N_{b}\right\}_{K_{a b}}\right)
\end{array}
$$

In this protocol, two participants $A$ and $B$ wish to establish a session key $K_{a b}$, with the help of a trusted server $S$. Initially, $A$ has a shared key $K_{a s}$ to communicate with $S$, and $B$ has a shared key $K_{b s}$ to communicate with $S$. In the 
first message, $A$ sends to $B$ his name $A$ and a nonce (fresh value) $N_{a}$. Then $B$ creates a nonce $N_{b}$ and sends to the server his own name $B$ and the encryption $\left\{A, N_{a}, N_{b}\right\}_{K_{b s}}$ of $A, N_{a}, N_{b}$ under the shared key $K_{b s}$. The server then creates the new (fresh) session key $K_{a b}$, and sends two encrypted messages to $A$. The first one $\left\{B, K_{a b}, N_{a}, N_{b}\right\}_{K_{a s}}$ gives the key $K_{a b}$ to $A$, together with $B$ 's name and the nonces (so that $A$ knows that the key is intended to communicate with $B$ ). The second message cannot be decrypted by $A$, so $A$ forwards it to $B$ (message 4). $B$ then obtains the session key $K_{a b}$. The second part of message $4,\left\{N_{b}\right\}_{K_{a b}}$, is used to check that $A$ and $B$ really use the same key $K_{a b}: B$ is going to check that he can decrypt the message with the newly received key. We encode only one principal playing each role, since others can be included in the attacker.

Message 1 is represented by the clause

$$
\operatorname{att}((\operatorname{host}(\mathrm{Kas}), \mathrm{Na}))
$$

meaning that the attacker gets host(Kas) and $\mathrm{Na}$ when intercepting message 1 . In this clause, the host name $A$ is represented by host(Kas). Indeed, the server has a table of pairs (host name, shared key to communicate between that host and the server), and this table can be conveniently represented by a constructor host. This constructor takes as parameter the secret key and returns the host name. So host names are written host $(k)$. The server can also match a term $\operatorname{host}(k)$ to find back the secret key. The attacker cannot do this operation (he does not have the key table), so there is no destructor clause for host. There is a constructor clause, since the attacker can build new hosts with new host keys:

$$
\operatorname{att}(k) \rightarrow \operatorname{att}(\operatorname{host}(k))
$$

Message 2 is represented by the clause:

$$
\operatorname{att}((a, n a)) \rightarrow \operatorname{att}((\text { host}(\mathrm{Kbs}), \operatorname{sencrypt}((a, n a, \mathrm{Nb}(a, n a)), \mathrm{Kbs})))
$$

The hypothesis means that a message $(a, n a)$ (corresponding to message 1 ) must be received before sending message 2 . It corresponds to the situation in which the attacker sends $(a, n a)$ to $B, B$ takes that for message 1 , and replies with message 2 , which is intercepted by the attacker. ( $a$ and $n a$ are variables since $B$ accepts any term instead of host(Kas) and $\mathrm{Na}$.) The nonce $N_{b}$ is represented by the function $\mathrm{Nb}(a, n a)$. Indeed, since a new name is created at each execution, names created after receiving different messages are different. This is modeled by considering names as functions of the messages previously received. This modeling is slightly weaker than creating a new name at each run of the protocol, but it is correct: if a secrecy property is proved in this model, then it is true [1]. The introduced function symbols will be called "name function symbols". (In message 1 , the fresh name $\mathrm{Na}$ is a constant because there are no previous messages on which it would depend.)

Message 3 is represented by the clause:

$$
\begin{aligned}
& \operatorname{att}((\operatorname{host}(k b s), \operatorname{sencrypt}((\operatorname{host}(k a s), n a, n b), k b s))) \\
& \rightarrow \operatorname{att}((\operatorname{sencrypt}((\operatorname{host}(k b s), \operatorname{Kab}(k a s, k b s, n a, n b), n a, n b), k a s),(\mathrm{Msg} 3) \\
& \operatorname{sencrypt}((\operatorname{host}(k a s), \operatorname{Kab}(k a s, k b s, n a, n b)), k b s)))
\end{aligned}
$$


using the same principles. At last, message 4 is represented by

$$
\operatorname{att}((\operatorname{sencrypt}((b, k, \mathrm{Na}, n b), \mathrm{Kas}), m b)) \rightarrow \operatorname{att}((m b, \operatorname{sencrypt}(n b, k)))
$$

The message sencrypt((host(Kas), $k$ ), Kbs) cannot be decrypted and checked by $A$, so it is a variable $m b$.

The goal of the protocol is to establish a secret shared key $K_{a b}$ between $A$ and $B$. If the key was a constant, say $\mathrm{K}_{\mathrm{ab}}$, then the non-derivability of the fact $\operatorname{att}\left(\mathrm{K}_{\mathrm{ab}}\right)$ from the Horn clauses presented so far would prove its secrecy. However, $K_{a b}$, as received by $A$, is a variable $k$. We therefore use the following fact. The key $K_{a b}$ received by $A$ is secret if and only if some constant secretA remains secret when $A$ sends it encrypted under the key $K_{a b}$. Thus, we add a clause that corresponds to the translation of an extra message of the protocol, Message 5 . $A \rightarrow B:\{\text { secretA }\}_{K_{a b}}$.

$$
\begin{aligned}
& \operatorname{att}((\operatorname{sencrypt}((\text { host }(\mathrm{Kbs}), k, \mathrm{Na}, n b), \mathrm{Kas}), m b)) \\
& \quad \rightarrow \operatorname{att}(\operatorname{sencrypt}(\operatorname{secret} \mathrm{A}, k))
\end{aligned}
$$

Now, the secrecy of the key $K_{a b}$ received by $A$ can be proved from the nonderivability of the fact att(secretA) from the set of clauses $\mathcal{R}_{\text {Primitives }} \cup \mathcal{R}_{\text {Prot }}$.

For the Yahalom protocol, the translation yields the union of the following sets of Horn clauses. $\mathcal{R}_{\text {CryptoConstr }}$ contains (sencrypt) and (host), $\mathcal{R}_{\text {CryptoDestr }}$ contains (sdecrypt), $\mathcal{R}_{\text {DataConstr }}$ contains the tuple construction and $\mathcal{R}_{\text {DataDestr }}$ the tuple projections (both not listed), and $\mathcal{R}_{\text {Prot }}$ contains (Msg1), (Msg2), (Msg3), (Msg4) and (Msg5) and three clauses translating the initial knowledge, $\operatorname{att}(\mathrm{N})$, att(host(Kas)), and att(host(Kbs)).

\section{The Resolution-Based Verification Algorithm}

To determine whether a fact is derivable from the clauses, we use a resolutionbased algorithm explained below. (We use the meta-variables $R, H, C, F$ for rule, hypothesis, conclusion, fact, respectively.)

The algorithm infers new clauses by resolution as follows: From two clauses $R=H \rightarrow C$ and $R^{\prime}=F \wedge H^{\prime} \rightarrow C^{\prime}$ (where $F$ is any hypothesis of $R^{\prime}$ ), it infers $R \circ_{F} R^{\prime}=\sigma H \wedge \sigma H^{\prime} \rightarrow \sigma C^{\prime}$, where $C$ and $F$ are unifiable and $\sigma$ is the most general unifier of $C$ and $F$. The clause $R \circ_{F} R^{\prime}$ is the combination of $R$ and $R^{\prime}$, where $R$ proves the hypothesis $F$ of $R^{\prime}$. The resolution is guided by a selection function sel. Namely, $\operatorname{sel}(R)$ returns a subset of the hypotheses of $R$, and the resolution step above is performed only when $\operatorname{sel}(R)=\emptyset$ and $F \in \operatorname{sel}\left(R^{\prime}\right)$.

We can use several selection functions. In this paper, we use:

$$
\operatorname{sel}(H \rightarrow C)=\left\{\begin{array}{l}
\emptyset \text { if all elements of } H \text { are of the form att }(x), x \text { variable } \\
\{F\} \text { where } F \neq \operatorname{att}(x), F \in H, \text { otherwise }
\end{array}\right.
$$


The algorithm uses the following optimizations:

- Decomposition of data constructors: decomp takes a clause and returns a set of clauses, built as follows. For each data constructor $f$, decomp replaces recursively all facts $\operatorname{att}\left(f\left(M_{1}, \ldots, M_{n}\right)\right)$ with $\operatorname{att}\left(M_{1}\right) \wedge \ldots \wedge \operatorname{att}\left(M_{n}\right)$. When such a fact is in the conclusion of a clause, $n$ clauses are created, with the same hypotheses and the conclusions att $\left(M_{1}\right), \ldots$, att $\left(M_{n}\right)$ respectively. With decomposition, the standard clauses for data constructors and projections can be removed. The soundness of this operation follows from the equivalence between $\operatorname{att}\left(f\left(M_{1}, \ldots, M_{n}\right)\right)$ and $\operatorname{att}\left(M_{1}\right) \wedge \ldots \wedge \operatorname{att}\left(M_{n}\right)$ in the presence of the clauses $\operatorname{att}\left(x_{1}\right) \wedge \ldots \wedge \operatorname{att}\left(x_{n}\right) \rightarrow \operatorname{att}\left(f\left(x_{1}, \ldots, x_{n}\right)\right)$ and $\operatorname{att}\left(f\left(x_{1}, \ldots, x_{n}\right)\right) \rightarrow \operatorname{att}\left(x_{i}\right)$ in $\mathcal{R}_{\text {DataConstr }}$ and $\mathcal{R}_{\text {DataDestr }}$.

- Elimination of duplicate hypotheses: elimdup takes a clause and returns the same clause after keeping only one copy of duplicate hypotheses.

- Elimination of hypotheses att $(x)$ : elimattx eliminates hypotheses att $(x)$ when $x$ does not appear elsewhere in the clause. Indeed, these hypotheses are always true, since the attacker has at least one term.

- Elimination of tautologies: elimtaut eliminates all tautologies (that is, clauses whose conclusion is already in the hypotheses) from a set of clauses.

- simplify groups all these simplifications. We extend elimdup and elimattx naturally to sets of clauses, and define simplify = elimtaut $\circ$ elimattx $\circ$ elimdup o decomp.

- condense $(\mathcal{R})$ applies simplify to each clause in $\mathcal{R}$ and then eliminates subsumed clauses. We say that $H_{1} \rightarrow C_{1}$ subsumes $H_{2} \rightarrow C_{2}$ if and only if there exists a substitution $\sigma$ such that $\sigma C_{1}=C_{2}$ and $\sigma H_{1} \subseteq H_{2}$. If $\mathcal{R}$ contains clauses $R$ and $R^{\prime}$, such that $R$ subsumes $R^{\prime}, R^{\prime}$ is eliminated. (In that case, $R$ can do all derivations that $R^{\prime}$ can do.)

We now define the algorithm saturate $\left(\mathcal{R}_{0}\right)$. Starting from condense $\left(\mathcal{R}_{0}\right)$, the algorithm adds clauses inferred by resolution with the selection function sel and condenses the set of clauses at each iteration step until a fixpoint is reached. When a fixpoint is reached, saturate $\left(\mathcal{R}_{0}\right)$ consists of the clauses $R$ in the fixpoint such that $\operatorname{sel}(R)=\emptyset$. By adapting the proof of [4] to this algorithm, it is easy to show that, for any $\mathcal{R}_{0}$ and any closed fact $F, F$ is derivable from $\mathcal{R}_{\mathrm{All}}=$ $\mathcal{R}_{0} \cup \mathcal{R}_{\text {DataConstr }} \cup \mathcal{R}_{\text {DataDestr }}$ if and only if it is derivable from $\operatorname{saturate}\left(\mathcal{R}_{0}\right) \cup$ $\mathcal{R}_{\text {DataConstr }}$.

Once the clauses of saturate $\left(\mathcal{R}_{0}\right)$ have been computed, we use a standard backward depth-first search to see if a fact can be derived from $\operatorname{saturate}\left(\mathcal{R}_{0}\right) \cup$ $\mathcal{R}_{\text {DataConstr }}$. Taking $\mathcal{R}_{0}=\mathcal{R}_{\text {CryptoConstr }} \cup \mathcal{R}_{\text {CryptoDestr }} \cup \mathcal{R}_{\text {Prot }}$, if att $(M)$ cannot be derived from saturate $\left(\mathcal{R}_{0}\right) \cup \mathcal{R}_{\text {DataConstr }}$ then the protocol preserves the secrecy of $M$.

The optimizations enable us to weaken the conditions that guarantee termination. For instance, the decomposition of data constructors makes it possible to obtain termination without tagging each data constructor application, while other constructors such as encryption must be tagged. In the Yahalom protocol, for example, without decomposition of data constructors, the algorithm would resolve the clause (Msg2 with itself, immediately yielding an infinite loop. 
Another consequence of the optimizations is that not all terms in a clause can be variables. Indeed, when $x \in\left\{x_{1}, \ldots, x_{n}\right\}$, the clause $\operatorname{att}\left(x_{1}\right) \wedge \ldots \wedge \operatorname{att}\left(x_{n}\right) \rightarrow$ $\operatorname{att}(x)$ is eliminated since it is a tautology. When $x \notin\left\{x_{1}, \ldots, x_{n}\right\}$, all hypotheses are eliminated, so the clause becomes att $(x)$ and all other clauses are eliminated since they are subsumed by att $(x)$, so the algorithm stops immediately: all facts can be derived. Thus, when $\operatorname{sel}(R)=\emptyset$, the conclusion of $R$ is not of the form att $(x)$. Therefore, the above selection function prevents resolution steps in which $\operatorname{att}(x)$ is unified with another fact (actually, with any other fact, which can lead to non-termination).

\section{Sufficient Conditions for Termination}

We are now collecting the formal properties of sets of Horn clauses (logic programs, or programs for short) that together entail termination. The properties for protocol programs hold for the translation of every protocol. The properties for plain protocol programs hold for the translation of protocols with a restriction on their cryptographic primitives and on their keys (this restriction is satisfied by many interesting protocols, including Yahalom for example). The properties for tagged protocol programs hold for the translation of those protocols after they have been tagged. The derivability problem for plain protocol programs is undecidable (as can be easily seen by a reduction to two-counter machines). The restriction to tagged programs makes the problem decidable, as will follow.

Given a clause $R$ of the form att $\left(M_{1}\right) \wedge \ldots \wedge \operatorname{att}\left(M_{n}\right) \rightarrow \operatorname{att}\left(M_{0}\right)$, we say that the terms $M_{0}, M_{1}, \ldots, M_{n}$ are the terms of $R$, and we denote the set of terms of $R$ by terms $(R)$.

Definition 2 (Protocol program). A protocol program is a set of clauses $\mathcal{R}_{\text {All }}=\mathcal{R}_{\text {Primitives }} \cup \mathcal{R}_{\text {Prot }}$ (where $\mathcal{R}_{\text {Primitives }}$ is a program for primitives) that comes with a finite set of closed terms $S_{0}$ such that:

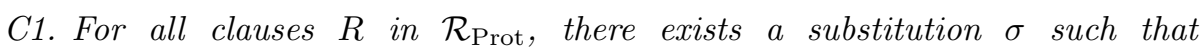
terms $(\sigma R) \subseteq S_{0}$.

C2. Every two subterms of terms in $S_{0}$ of the form a(...) with the same name function symbol a are identical.

C3. The second argument of pencrypt in $S_{0}$ is of the form $\mathrm{pk}(M)$ for some $M$.

The terminology "argument of $f$ in $S_{0}$ " refers to a term $M$ such that $f(\ldots, M, \ldots)$ is a subterm of a term in $S_{0}$. To see why these conditions are satisfied by a translation of a protocol, let us consider the intended messages of the protocol. These are the exchanged messages when the attacker does not intervene and when there is no unexpected interaction between sessions of the protocol. We denote by $M_{1}, \ldots, M_{k}$ the closed terms corresponding to these messages. Each participant does not necessarily have a full view of the messages he receives; instead, he accepts all messages that are instances of patterns representing the information he can check. The terms $M_{1}, \ldots, M_{k}$ are particular instances 
of these patterns. So the protocol is represented by clauses $R$ such that there exists $\sigma$ such that terms $(\sigma R) \subseteq\left\{M_{1}, \ldots, M_{k}\right\}$. Defining $S_{0}=\left\{M_{1}, \ldots, M_{k}\right\} \cup S_{\text {Init }}$, we obtain $\mathrm{C} 1$.

For instance, the intended messages for the Yahalom protocol are

$$
\begin{aligned}
& M_{1}=(\text { host }(\mathrm{Kas}), \mathrm{Na}) \\
& M_{2}=\left(\operatorname{host}(\mathrm{Kbs}), \operatorname{sencrypt}\left(\left(\operatorname{host}(\mathrm{Kas}), \mathrm{Na}, M_{N_{b}}\right), \mathrm{Kbs}\right)\right) \\
& M_{3}=\left(\operatorname{sencrypt}\left(\left(\operatorname{host}(\mathrm{Kbs}), M_{K}, \mathrm{Na}, M_{N_{b}}\right), \mathrm{Kas}\right), \operatorname{sencrypt}\left(\left(\operatorname{host}(\mathrm{Kas}), M_{K}\right), \mathrm{Kbs}\right)\right) \\
& M_{4}=\left(\operatorname{sencrypt}\left(\left(\operatorname{host}(\mathrm{Kas}), M_{K}\right), \mathrm{Kbs}\right), \operatorname{sencrypt}\left(M_{N_{b}}, M_{K}\right)\right) \\
& M_{5}=\operatorname{sencrypt}\left(\operatorname{secret} \mathrm{A}, M_{K}\right)
\end{aligned}
$$

with $M_{N_{b}}=\mathrm{Nb}$ (host(Kas), $\mathrm{Na}$ ) and $M_{K}=\mathrm{Kab}\left(\mathrm{Kas}, \mathrm{Kbs}, \mathrm{Na}, M_{N_{b}}\right)$. It is easy to check that the clauses (Msg1)-(Msg5) satisfy the condition C1.

Condition $\mathrm{C} 2$ models that each name function symbol is created at a unique occurrence in the protocol. Condition C3 means that, in its intended behaviour, the protocol uses public-key encryption only with public keys.

Definition 3 (Plain protocol program). A plain protocol program is a protocol program $\mathcal{R}_{\mathrm{All}}$ with associated set of closed terms $S_{0}$, such that:

C4. The only constructors and destructors are those of Figure 1, plus host.

C5. The arguments of $\mathrm{pk}$ and host in $S_{0}$ are atomic constants.

Condition C5 essentially means that the protocol only uses pairs of atomic keys for public key cryptography, and atomic keys for long-term secret keys.

Tagging a protocol is a simple syntactic annotation of messages. We add a tag to each application of a primitive sencrypt, pencrypt, sign, nmrsign, hash, mac, such that two applications of the same primitive with the same tag have the same parameters. For example, after tagging the Yahalom protocol becomes:

$$
\begin{aligned}
& \text { Message 1. } A \rightarrow B:\left(A, N_{a}\right) \\
& \text { Message 2. } B \rightarrow S:\left(B,\left\{\mathrm{c}_{1}, A, N_{a}, N_{b}\right\}_{K_{b s}}\right) \\
& \text { Message 3. } S \rightarrow A:\left(\left\{\mathrm{c}_{2}, B, K_{a b}, N_{a}, N_{b}\right\}_{K_{a s}},\left\{\mathrm{c}_{3}, A, K_{a b}\right\}_{K_{b s}}\right) \\
& \text { Message 4. } A \rightarrow B:\left(\left\{\mathrm{c}_{3}, A, K_{a b}\right\}_{K_{b s}},\left\{\mathrm{c}_{4}, N_{b}\right\}_{K_{a b}}\right)
\end{aligned}
$$

If the original protocol translates to a plain protocol program, its tagged version translates to a tagged protocol program, as defined below.

Definition 4 (Tagged protocol program). A tagged protocol program is a plain protocol program $\mathcal{R}_{\text {All }}$ with associated set of closed terms $S_{0}$ such that:

C6. If $f \in$ \{sencrypt, pencrypt, sign, nmrsign, hash, mac $\}$ occurs in a term in $S_{0}$ or in terms $(R)$ for $R \in \mathcal{R}_{\text {Prot }}$, then its first argument is the tuple (c, $\left.M_{1}, \ldots, M_{n}\right)$ for some constant c and terms $M_{1}, \ldots, M_{n}$.

C7. Every two subterms of terms in $S_{0}$ of the form $f((\mathrm{c}, \ldots), \ldots)$ with the same primitive $f \in\{$ sencrypt, pencrypt, sign, nmrsign, hash, mac $\}$ and the same tag c are identical. 
The condition that constant tags appear in $\operatorname{terms}(R)$ (Condition C6) means that honest protocol participants always check the tags of received messages (something that the informal description of a tagged protocol leaves implicit) and send tagged terms. The condition also expresses that the initial knowledge of the attacker consists of tagged terms.

\section{Termination Proof}

Instead of giving the termination proof in one big step, we first consider a special case (Section 5.1), and then describe the modification of the first proof that yields the proof for the general case (Section 5.2).

The special case is defined in terms of the sets Params $\mathrm{pk}$ and Params host of arguments of pk resp. host in $S_{0}$, namely by the condition that these sets each have at most one element.

This restriction is meaningful in terms of models of protocols: it corresponds to merging several keys. In the example of the Yahalom protocol, this means that, in the clauses, the keys $\mathrm{Kas}$ and $\mathrm{Kbs}$ should be replaced with a single key, $\mathrm{k}_{0}$ (so the host names $A=\operatorname{host}(\mathrm{Kas})$ and $B=\operatorname{host}(\mathrm{Kbs})$ are replaced with a single name host $\left.\left(\mathrm{k}_{0}\right)\right)$. When studying secrecy, merging all keys of honest hosts in this way helps to model cases in which one host plays several roles in the protocol. The secrecy for the clauses with merged keys implies secrecy for the protocol without merged keys. However, this merging is not acceptable for authenticity 5]. This is why we also consider the general case in Section 5.2.

\subsection{The Special Case of One Key}

We now define weakly tagged programs by the conditions that we use in the first termination proof. In the special case, these conditions are strictly more general than tagged protocol programs. This plays a role to deduce termination for protocols that are not explicitly tagged (see Remark 1).

A term is said to be non-data when it is not of the form $f(\ldots)$ with $f$ in DataConstr. The set $\operatorname{sub}(S)$ contains the subterms of terms in the set $S$.

The set tagGen contains the non-variable non-data subterms of terms of clauses in $\mathcal{R}_{\text {Prot }}$ and of terms $M_{1}, \ldots, M_{n}$ in clauses of the form $\operatorname{att}\left(f\left(M_{1}, \ldots, M_{n}\right)\right) \wedge \operatorname{att}\left(x_{1}\right) \wedge \ldots \wedge \operatorname{att}\left(x_{m}\right) \rightarrow \operatorname{att}(x)$ in condense $\left(\mathcal{R}_{\text {CryptoDestr }}\right)$ (this is the form required in W1 below). This set summarizes the terms that appear in the clauses and that should be tagged.

Definition 5 (Weakly tagged programs). A program $\mathcal{R}_{\mathrm{All}}$ of the form $\mathcal{R}_{\text {All }}=\mathcal{R}_{\text {Primitives }} \cup \mathcal{R}_{\text {Prot }}$ (where $\mathcal{R}_{\text {Primitives }}$ is a program for primitives) is weakly tagged if there exists a finite set of closed terms $S_{0}$ such that:

$W 1$. All clauses in the set $\mathcal{R}_{\text {CryptoDestr }}^{\prime}=$ condense $\left(\mathcal{R}_{\text {CryptoDestr }}\right)$ are of the form

$$
\operatorname{att}\left(f\left(M_{1}, \ldots, M_{n}\right)\right) \wedge \operatorname{att}\left(x_{1}\right) \wedge \ldots \wedge \operatorname{att}\left(x_{m}\right) \rightarrow \operatorname{att}(x)
$$

where $f \in$ CryptoConstr, $x$ is one of $M_{1}, \ldots, M_{n}$, and $f\left(M_{1}, \ldots, M_{n}\right)$ is more general than every term of the form $f(.$.$) in \operatorname{sub}\left(S_{0}\right)$. 
W2. For all clauses $R$ in $\mathcal{R}_{\text {Prot, }}$ there exists a substitution $\sigma$ such that terms $(\sigma R) \subseteq S_{0}$.

W3. If two terms $M_{1}$ and $M_{2}$ in tagGen unify, $N_{1}$ is an instance of $M_{1}$ in $\operatorname{sub}\left(S_{0}\right)$, and $N_{2}$ is an instance of $M_{2}$ in $\operatorname{sub}\left(S_{0}\right)$, then $N_{1}=N_{2}$.

Condition W3 is the key of the termination proof. We are going to show the following invariant: all terms in the generated clauses are instances of terms in tagGen and have instances in $\operatorname{sub}\left(S_{0}\right)$. This condition makes it possible to prove that, when unifying two terms satisfying the invariant, the result of the unification also satisfies the invariant; this is because the instances in $\operatorname{sub}\left(S_{0}\right)$ of those two terms are in fact equal. Condition W1 guarantees that this continues to hold if only one of the two terms satisfies the invariant and the other stems from a clause in $\mathcal{R}_{\text {CryptoDestr }}^{\prime}$.

Proposition 1. A tagged protocol program where Params Post $_{\text {and Params }}$ Pk each have at most one element, is weakly tagged.

Proof. For condition W1, the clauses for sdecrypt, pdecrypt, and getmessage are:

$$
\begin{aligned}
& \operatorname{att}(\operatorname{sencrypt}(x, y)) \wedge \operatorname{att}(y) \rightarrow \operatorname{att}(x) \\
& \operatorname{att}(\operatorname{pencrypt}(x, \operatorname{pk}(y))) \wedge \operatorname{att}(y) \rightarrow \operatorname{att}(x) \\
& \operatorname{att}(\operatorname{sign}(x, y)) \rightarrow \operatorname{att}(x)
\end{aligned}
$$

and they satisfy condition W1 provided that all public-key encryptions in $S_{0}$ are of the form pencrypt $\left(M_{1}, \operatorname{pk}\left(M_{2}\right)\right.$ ) (that is C3). The clauses for checksignature and nmrchecksign are

$$
\begin{aligned}
& \operatorname{att}(\operatorname{sign}(x, y)) \wedge \operatorname{att}(\operatorname{pk}(y)) \rightarrow \operatorname{att}(x) \\
& \operatorname{att}(\operatorname{nmrsign}(x, y)) \wedge \operatorname{att}(\operatorname{pk}(y)) \wedge \operatorname{att}(x) \rightarrow \operatorname{att}(\operatorname{true})
\end{aligned}
$$

These two clauses are subsumed respectively by the clauses for getmessage (given above) and true (which is simply att(true) since true is a zero-ary constructor), so they are eliminated by condense, i.e., they are not in $\mathcal{R}_{\text {CryptoDestr. }}^{\prime}$ (This is important, because they do not satisfy condition W1.)

Condition W2 is identical to condition $\mathrm{C} 1$. We now prove condition W3. Let

$$
\begin{aligned}
S_{1}=\{ & f\left(\left(\mathrm{c}_{i}, x_{1}, \ldots, x_{n}\right), x_{2}^{\prime}, \ldots, x_{n^{\prime}}^{\prime}\right) \mid \\
& f \in\{\text { sencrypt, pencrypt, sign, nmrsign, hash, mac }\}\} \\
\cup & \left\{a\left(x_{1}, \ldots, x_{n}\right) \mid a \text { name function symbol }\right\} \\
\cup & \{\operatorname{pk}(x), \operatorname{host}(x)\} \cup\{c \mid c \text { atomic constant }\}
\end{aligned}
$$

By condition $\mathrm{C} 4$, the only term in tagGen that comes from clauses of $\mathcal{R}_{\text {CryptoDestr }}^{\prime}$ is $\mathrm{pk}(x)$. Using condition C6, all terms in tagGen are instances of terms in $S_{1}$ (noticing that tagGen does not contain variables). Using conditions C2, C5, C7,

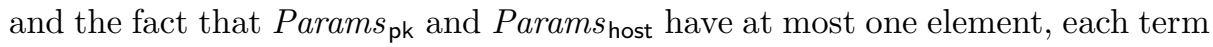
in $S_{1}$ has at most one instance in $\operatorname{sub}\left(S_{0}\right)$. 
If $M_{1}$ and $M_{2}$ in tagGen unify, they are both instances of the same element $M^{\prime}$ in $S_{1}$ (since different elements of $S_{1}$ do not unify with each other). Let $N_{1}$ and $N_{2}$ be any instances of $M_{1}$ and $M_{2}$ (respectively) in $\operatorname{sub}\left(S_{0}\right)$. Then $N_{1}$ and $N_{2}$ are instances of $M^{\prime} \in S_{1}$ in $\operatorname{sub}\left(S_{0}\right)$ so $N_{1}=N_{2}$. Thus we obtain W3.

Remark 1. The Yahalom protocol is in fact weakly tagged without explicitly adding constant tags (after merging the keys Kas and Kbs). Indeed, since different encryptions in the protocol have a different arity, we can take sencrypt $\left(\left(x_{1}\right.\right.$, $\left.\left.\ldots, x_{n}\right), x^{\prime}\right)$ in $S_{1}$ in the proof above, and use the same reasoning as above to prove the condition W3. This shows both that type flaws cannot happen in the original protocol, and that the algorithm also terminates on the original protocol. We can say that the protocol is "implicitly tagged": the arity replaces the tag. This situation happens in some other examples, and can partly explain why the algorithm often terminates even for protocols without explicit tags.

A term is top-tagged when it is an instance of a term in tagGen. Intuitively, referring to the case of explicit constant tags, top-tagged terms are terms whose top function symbol is tagged. A term is fully tagged when all its non-variable non-data subterms are top-tagged.

We next show the invariant that all terms in the generated clauses are nondata, fully tagged, and have instances in $\operatorname{sub}\left(S_{0}\right)$. Using this invariant, we show that the size of an instance in $\operatorname{sub}\left(S_{0}\right)$ of a clause obtained by resolution from $R$ and $R^{\prime}$ is smaller than the size of an instance of $R$ or $R^{\prime}$ in $\operatorname{sub}\left(S_{0}\right)$. This implies the termination of the algorithm.

Let us define the size of a term $M$, size $(M)$, as usual, and the size of a clause by $\operatorname{size}\left(\operatorname{att}\left(M_{1}\right) \wedge \ldots \wedge \operatorname{att}\left(M_{n}\right) \rightarrow \operatorname{att}(M)\right)=\operatorname{size}\left(M_{1}\right)+\ldots+\operatorname{size}\left(M_{n}\right)+\operatorname{size}(M)$. The hypotheses of clauses form a multiset, so when we compute $\operatorname{size}(\sigma R)$ and the substitution $\sigma$ maps several hypotheses to the same fact, this fact is counted several times in size. Intuitively, the size of clauses can increase during resolution, because the unification can instantiate terms. However, the size of their corresponding closed instance in $\operatorname{sub}\left(S_{0}\right)$ decreases.

Proposition 2. Assuming a weakly tagged program (Definition 5) and $\mathcal{R}_{0}=$ $\mathcal{R}_{\text {CryptoConstr }} \cup \mathcal{R}_{\text {CryptoDestr }} \cup \mathcal{R}_{\text {Prot }}$, the computation of saturate $\left(\mathcal{R}_{0}\right)$ terminates.

Proof. We show by induction that all rules $R$ generated from $\mathcal{R}_{0}$ either are in $\mathcal{R}_{\text {CryptoConstr }} \cup \mathcal{R}_{\text {CryptoDestr }}^{\prime}$, or are such that the terms of $R$ are non-data, fully tagged, and mapped to $\operatorname{sub}\left(S_{0}\right)$ by a substitution $\sigma$, i.e., $\operatorname{terms}(\sigma R) \subseteq \operatorname{sub}\left(S_{0}\right)$.

First, we can easily show that all rules in condense $\left(\mathcal{R}_{0}\right)$ satisfy this property.

If we combine by resolution two rules in $\mathcal{R}_{\text {CryptoConstr }} \cup \mathcal{R}_{\text {CryptoDestr }}^{\prime}$, we in

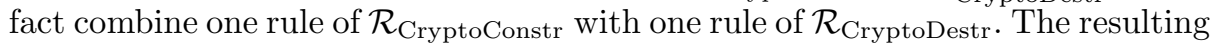
rule is a tautology by condition $\mathrm{W} 1$, so it is eliminated immediately.

Otherwise, we combine by resolution a rule $R$ such that the terms of $R$ are non-data and fully tagged, and there exists a substitution $\sigma$ such that terms $(\sigma R) \subseteq \operatorname{sub}\left(S_{0}\right)$, with a rule $R^{\prime}$ such that one of 1., 2., or 3. holds. 
1. The terms of $R^{\prime}$ are non-data and fully tagged, there exists a substitution $\sigma^{\prime}$ such that terms $\left(\sigma^{\prime} R^{\prime}\right) \subseteq \operatorname{sub}\left(S_{0}\right)$, and $\operatorname{sel}\left(R^{\prime}\right)=\emptyset($ in which case $\operatorname{sel}(R) \neq \emptyset)$.

2. $R^{\prime} \in \mathcal{R}_{\text {CryptoConstr }}$.

3. $R^{\prime} \in \mathcal{R}_{\text {CryptoDestr }}^{\prime}$.

Let $R^{\prime \prime}$ be the rule obtained by resolution of $R$ and $R^{\prime}$. We show that the terms of $R^{\prime \prime}$ are fully tagged, and there exists a substitution $\sigma^{\prime \prime}$ such that terms $\left(\sigma^{\prime \prime} R^{\prime \prime}\right) \subseteq$ $\operatorname{sub}\left(S_{0}\right)$ and size $\left(\sigma^{\prime \prime} R^{\prime \prime}\right)<\operatorname{size}(\sigma R)$.

Let $M_{0}, \ldots, M_{n}$ be the terms of $R$, att $\left(M_{0}\right)$ being the atom of $R$ on which we resolve. In all cases, the terms of $R^{\prime}$ are $M^{\prime}, x_{1}, \ldots, x_{n^{\prime}}$, the variables $x_{1}, \ldots, x_{n^{\prime}}$ occur in $M^{\prime}$ and are pairwise distinct variables, and att $\left(M^{\prime}\right)$ is the atom of $R^{\prime}$ on which we resolve. (In case 1, because $\operatorname{sel}\left(R^{\prime}\right)=\emptyset$ and by the optimizations elimattx and elimdup; in case 2. by definition of constructor rules; in case 3 . by W1.) The terms $M_{0}$ and $M^{\prime}$ unify, let $\sigma_{u}$ be their most general unifier. Then the terms of $R^{\prime \prime}$ are $\sigma_{u} x_{1}, \ldots, \sigma_{u} x_{n^{\prime}}, \sigma_{u} M_{1}, \ldots, \sigma_{u} M_{n}$. By the choice of the selection function, the terms $M_{0}$ and $M^{\prime}$ are not variables.

We know that $\sigma M_{0}, \ldots, \sigma M_{n}$ are in $\operatorname{sub}\left(S_{0}\right)$. We show that there exists $\sigma^{\prime}$ such that $\sigma M_{0}=\sigma^{\prime} M^{\prime}$.

- In case 1 there exists $\sigma^{\prime}$ such that $\sigma^{\prime} M^{\prime} \in \operatorname{sub}\left(S_{0}\right)$. The terms $M_{0}$ and $M^{\prime}$ are non-data fully tagged, so all their non-variable non-data subterms are top-tagged. In particular, since they are not variables, $M_{0}$ and $M^{\prime}$ themselves are top-tagged, i.e., $M_{0}$ is an instance of some $N_{0} \in \operatorname{tag} G e n$ and $M^{\prime}$ is an instance of some $N_{0}^{\prime} \in$ tagGen. Since $M_{0}$ and $M^{\prime}$ unify, so do $N_{0}$ and $N_{0}^{\prime}$, $\sigma^{\prime} M^{\prime}$ is an instance of $N_{0}^{\prime}$ in $\operatorname{sub}\left(S_{0}\right), \sigma M_{0}$ is an instance of $N_{0}$ in $\operatorname{sub}\left(S_{0}\right)$, so by condition $\mathrm{W} 3, \sigma^{\prime} M^{\prime}=\sigma M_{0}$.

- In case 2, $M^{\prime}$ is of the form $f\left(x_{1}, \ldots, x_{n^{\prime}}\right)$. Since $M_{0}$ is not a variable and unifies with $M^{\prime}, M_{0}$ has root symbol $f$, so $\sigma M_{0}$ is an instance of $M^{\prime}$.

- In case 3, by condition W1, $M^{\prime}$ is more general than every term in $\operatorname{sub}\left(S_{0}\right)$ with the same root symbol, hence the instance $\sigma M_{0}$ of the term $M_{0}$ that is unifiable with $M^{\prime}$ and thus has the same root symbol.

The substitution equal to $\sigma$ on the variables of $R$ and to $\sigma^{\prime}$ on the variables of $R^{\prime}$ is then a unifier of $M_{0}$ and $M^{\prime}$. Since $\sigma_{u}$ is the most general unifier, there exists $\sigma^{\prime \prime}$ such that $\sigma^{\prime \prime} \sigma_{u}$ is equal to $\sigma$ on the variables of $R$, and to $\sigma^{\prime}$ on the variables of $R^{\prime}$. Thus the terms of $\sigma^{\prime \prime} R^{\prime \prime}$ are $\sigma^{\prime} x_{1}, \ldots, \sigma^{\prime} x_{n^{\prime}}, \sigma M_{1}, \ldots, \sigma M_{n}$. The terms $\sigma^{\prime} x_{1}, \ldots, \sigma^{\prime} x_{n^{\prime}}$ are subterms of $\sigma^{\prime} M^{\prime}=\sigma M_{0}$ which is in $\operatorname{sub}\left(S_{0}\right)$, so they are also in $\operatorname{sub}\left(S_{0}\right)$. So all terms of $\sigma^{\prime \prime} R^{\prime \prime}$ are in $\operatorname{sub}\left(S_{0}\right)$.

Moreover, size $\left(\sigma^{\prime \prime} R^{\prime \prime}\right)<\operatorname{size}\left(\sigma^{\prime} R^{\prime}\right)$. Indeed, $x_{1}, \ldots, x_{n^{\prime}}$ occur in $M^{\prime}$ and are different variables. So $\sigma^{\prime} x_{1}, \ldots, \sigma^{\prime} x_{n^{\prime}}$ are disjoint subterms of $\sigma^{\prime} M^{\prime}$, and $M^{\prime}$ does not consist of only a variable, so size $\left(\sigma^{\prime} x_{1}\right)+\ldots+\operatorname{size}\left(\sigma^{\prime} x_{n^{\prime}}\right)<\operatorname{size}\left(\sigma^{\prime} M^{\prime}\right)=$ $\operatorname{size}\left(\sigma M_{0}\right)$, and size $\left(\sigma^{\prime \prime} R^{\prime \prime}\right)<\operatorname{size}\left(\sigma M_{0}\right)+\ldots+\operatorname{size}\left(\sigma M_{n}\right)=\operatorname{size}(\sigma R)$.

We show that the terms of $R^{\prime \prime}$ are fully tagged.

- In case 1 since $\sigma_{u}$ is the most general unifier of fully tagged terms, we can show that, for all $x, \sigma_{u} x$ is fully tagged, so for all fully tagged terms $M$, we can show that $\sigma_{u} M$ is fully tagged, so the terms of $R^{\prime \prime}$ are fully tagged. 
- In case 2, for $x$ among $x_{1}, \ldots, x_{n^{\prime}}, \sigma_{u} x$ is a subterm of $M_{0}$, so is fully tagged. The terms $\sigma_{u} M_{1}, \ldots, \sigma_{u} M_{n}$ are equal to $M_{1}, \ldots, M_{n}$, also fully tagged.

- In case 3, $M^{\prime}=f\left(M_{1}^{\prime}, \ldots, M_{m}^{\prime}\right)$ and $M_{0}=f\left(M_{1}^{\prime \prime}, \ldots, M_{m}^{\prime \prime}\right)$, so $\sigma_{u}$ is also the most general unifier of the pairs $\left(M_{1}^{\prime}, M_{1}^{\prime \prime}\right), \ldots,\left(M_{m}^{\prime}, M_{m}^{\prime \prime}\right)$ of fully tagged terms. So we conclude as in case 1

Finally, the terms of $R^{\prime \prime}$ are fully tagged, terms $\left(\sigma^{\prime \prime} R^{\prime \prime}\right) \subseteq \operatorname{sub}\left(S_{0}\right)$, and $\operatorname{size}\left(\sigma^{\prime \prime} R^{\prime \prime}\right)<\operatorname{size}(\sigma R)$.

Then it is easy to show that all rules $R_{\mathrm{S}} \in \operatorname{simplify}\left(R^{\prime \prime}\right)$ obtained after simplification of $R^{\prime \prime}$ have non-data fully tagged terms and satisfy terms $\left(\sigma^{\prime \prime} R_{\mathrm{s}}\right) \subseteq$ $\operatorname{sub}\left(S_{0}\right)$, and size $\left(\sigma^{\prime \prime} R_{\mathrm{s}}\right)<\operatorname{size}(\sigma R)$. Indeed, all rules in decomp $\left(R^{\prime \prime}\right)$ satisfy this property. (The decomposition of data constructors transforms fully tagged terms into non-data fully tagged terms.) This property is preserved by elimdup and elimattx.

Therefore, for all generated rules $R$, there exists $\sigma$ such that size $(\sigma R)$ is smaller than the maximum initial value of size $(\sigma R)$ for a rule of the protocol. There is a finite number of such rules (since size $(R) \leq \operatorname{size}(\sigma R)$ ). So the algorithm terminates.

The termination of the backward depth-first search for closed facts is easy to show, for example by a proof similar to that of [4]. Essentially, the size of the goal decreases, because the size of the hypotheses of each clause is smaller than the size of the conclusion. (Recall that all terms of hypotheses of clauses of saturate $\left(\mathcal{R}_{0}\right) \cup \mathcal{R}_{\text {DataConstr }}$ are variables that occur in the conclusion.) So we obtain:

Theorem 1. The resolution-based verification algorithm terminates for weakly tagged programs and closed facts.

As a corollary, by Proposition[1, we obtain the same result for tagged protocol programs, when Params host and Params

\subsection{Handling Several Keys}

The extension to several arguments of pk or of host requires an additional step. We define a homomorphism $h$ from terms to terms that replaces all elements

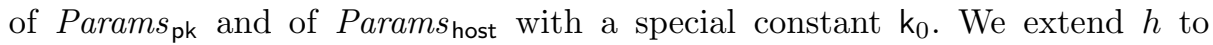
facts, clauses, and sets of clauses naturally. For the protocol program $h\left(\mathcal{R}_{\text {Prot }}\right)$, Params $_{\mathrm{pk}}$ and Params host $_{\text {h }}$ each have at most one element. So by Proposition [1] when $\mathcal{R}_{\text {Prot }}$ is a tagged protocol program, $h\left(\mathcal{R}_{\text {Prot }}\right)$ is a weakly tagged program.

Let $\mathcal{R}_{\text {Prot }}$ be any program such that $h\left(\mathcal{R}_{\text {Prot }}\right)$ is a weakly tagged program. We consider a "less optimized algorithm" in which elimination of duplicate hypotheses and of tautologies are performed only for facts of the form $\operatorname{att}(x)$ and elimination of subsumed clauses is performed only for the condensing of rules of $\mathcal{R}_{\text {CryptoDestr. }}$. We observe that Theorem 1 holds also for the less optimized algorithm, with the same proof, so this algorithm terminates on $h\left(\mathcal{R}_{\text {Prot }}\right)$. All resolution steps possible for the less optimized algorithm applied to $\mathcal{R}_{\text {Prot }}$ are 
possible for the less optimized algorithm applied to $h\left(\mathcal{R}_{\text {Prot }}\right)$ as well (more terms are unifiable, and the remaining optimizations of the less optimized algorithm commute with the application of $h$ ). Then the less optimized algorithm terminates on $\mathcal{R}_{\text {Prot }}$. We can show that then the original, fully optimized algorithm also terminates.

In particular, the algorithm terminates for all tagged protocol programs and for implicitly tagged protocols, such as the Yahalom protocol without tags by Remark 1 .

Theorem 2. The resolution-based verification algorithm terminates for tagged protocol programs and closed facts.

We recall that a tagged protocol program may be obtained by translating a protocol after tagging, and that the algorithm checks the non-derivability of the closed fact att $(M)$, which shows the secrecy of the message $M$.

Although, for tagged protocols, the worst-case complexity of the algorithm is exponential (we did not detail this result by lack of space), it is quite efficient in practice. It remains to be seen whether there exists a smaller class containing most interesting examples, and for which the algorithm is polynomial.

\section{Related Work}

The verification problem of cryptographic protocols is undecidable [13], so one either restricts the problem, or approximates it.

Decision procedures have been published for restricted cases. In the case of a bounded number of sessions, for protocols using public-key cryptography with atomic keys and shared-key cryptography, protocol insecurity is NPcomplete [23], and decisions procedures appear in [11 1923. When messages are bounded and no nonces are created, secrecy is DEXPTIME-complete [13]. Strong syntactic restrictions on protocols also yield decidability: 10] for an extension of ping-pong protocols, [3] with a bound on the number of parallel sessions, and restricted matching on incoming messages (in particular, this matching should be linear and independent of previous messages). Model-checking also provides a decision technique for a bounded number of sessions [18] (with additional conditions). It has been extended, with approximations, to an unbounded number of sessions using data independence techniques [67/22], for sequential runs, or when the agents are "factorisable". (Essentially, a single run of the agent has to be split into several runs, such that each run contains only one fresh value.)

On the other hand, some analyses terminate for all protocols, but at the cost of approximations. For instance, control-flow analysis [20] runs in cubic time, but does not preserve relations between components of messages, hence introduces an important approximation. Interestingly, the proof that control flow analysis runs in cubic time also relies on the study of a particular class of Horn clauses. Techniques using tree automata [15] and rank functions [17] also provide a terminating but approximate analysis. Moreover, the computation algorithm of rank functions assumes atomic keys. 
It has been shown in [16] that tagging prevents type flaw attacks. It may be possible to infer from [16] that the depth of closed terms can be bounded in the search for an attack. This yields the decidability by exhaustive search, but does not imply the termination of our algorithm (in particular, because clauses can have an unbounded number of hypotheses, so there is an infinite number of clauses with a bounded term depth).

As for the approach based on Horn clauses, Weidenbach [24] already gave conditions under which his algorithm terminates. These conditions may give some idea of why the algorithm terminates on protocols. They do not seem to apply to many examples of cryptographic protocols.

Other techniques such as theorem proving [21] in general require human intervention, even if some cases can be proved automatically 912. In general, typing [1,14] requires human intervention in the form of type annotations, that can be automatically checked. The idea of tagging already appears in 14] in a different context (tagged union types).

\section{Conclusion}

We have given the theory behind an experimental observation: tagging a protocol enforces the termination of the resolution-based verification technique used. Our work has an obvious consequence to protocol design, namely when one agrees that a design choice in view of a-posteriori verification is desirable.

Our termination result for weakly tagged protocols explains only in part another experimental observation, namely the termination for protocols without explicit tags. Although many of those are weakly tagged, some of them are not (for instance, the Needham-Schroeder public key protocol). The existence of a termination condition that applies also to those cases is open.

\section{References}

1. M. Abadi and B. Blanchet. Analyzing Security Protocols with Secrecy Types and Logic Programs. In 29th ACM Symp. on Principles of Programming Languages (POPL'02), pages 33-44, Jan. 2002.

2. M. Abadi and R. Needham. Prudent engineering practice for cryptographic protocols. IEEE Transactions on Software Engineering, 22(1):6-15, Jan. 1996.

3. R. Amadio and W. Charatonik. On Name Generation and Set-Based Analysis in the Dolev-Yao Model. In CONCUR'02 - Concurrency Theory, volume 2421 of LNCS, pages 499-514. Springer, Aug. 2002.

4. B. Blanchet. An Efficient Cryptographic Protocol Verifier Based on Prolog Rules. In 14th IEEE Computer Security Foundations Workshop (CSFW-14), pages 82-96, June 2001.

5. B. Blanchet. From Secrecy to Authenticity in Security Protocols. In 9th Internat. Static Analysis Symposium (SAS'02), volume 2477 of LNCS, pages 342-359. Springer, Sept. 2002.

6. P. Broadfoot, G. Lowe, and B. Roscoe. Automating Data Independence. In 6th European Symp. on Research in Computer Security (ESORICS'00), volume 1895 of LNCS, pages 175-190. Springer, Oct. 2000. 
7. P. J. Broadfoot and A. W. Roscoe. Capturing Parallel Attacks within the Data Independence Framework. In 15th IEEE Computer Security Foundations Workshop (CSFW'02), pages 147-159, June 2002.

8. M. Burrows, M. Abadi, and R. Needham. A Logic of Authentication. Proceedings of the Royal Society of London A, 426:233-271, 1989.

9. E. Cohen. TAPS: A First-Order Verifier for Cryptographic Protocols. In 13th IEEE Computer Security Foundations Workshop (CSFW-13), pages 144-158, 2000.

10. H. Comon, V. Cortier, and J. Mitchell. Tree Automata with One Memory, Set Constraints, and Ping-Pong Protocols. In Automata, Languages and Programming, 28th Internat. Colloq., ICALP'01, volume 2076 of LNCS, pages 682-693. Springer, July 2001.

11. R. Corin and S. Etalle. An Improved Constraint-Based System for the Verification of Security Protocols. In 9th Internat. Static Analysis Symposium (SAS'02), volume 2477 of $L N C S$, pages 326-341. Springer, Sept. 2002.

12. V. Cortier, J. Millen, and H. Rueß. Proving secrecy is easy enough. In 14 th IEEE Computer Security Foundations Workshop (CSFW-14), pages 97-108, June 2001.

13. N. A. Durgin, P. D. Lincoln, J. C. Mitchell, and A. Scedrov. Undecidability of bounded security protocols. In Workshop on Formal Methods and Security Protocols (FMSP'99), July 1999.

14. A. Gordon and A. Jeffrey. Authenticity by Typing for Security Protocols. In 14th IEEE Computer Security Foundations Workshop (CSFW-14), pages 145-159, June 2001.

15. J. Goubault-Larrecq. A Method for Automatic Cryptographic Protocol Verification (Extended Abstract), invited paper. In 5th Internat. Workshop on Formal Methods for Parallel Programming: Theory and Applications (FMPPTA'00), volume 1800 of $L N C S$, pages 977-984. Springer, May 2000.

16. J. Heather, G. Lowe, and S. Schneider. How to Prevent Type Flaw Attacks on Security Protocols. In 13th IEEE Computer Security Foundations Workshop (CSFW13), pages 255-268, July 2000.

17. J. Heather and S. Schneider. Towards automatic verification of authentication protocols on an unbounded network. In 13th IEEE Computer Security Foundations Workshop (CSFW-13), pages 132-143, July 2000.

18. G. Lowe. Breaking and Fixing the Needham-Schroeder Public-Key Protocol using FDR. In Tools and Algorithms for the Construction and Analysis of Systems, volume 1055 of LNCS, pages 147-166. Springer, 1996.

19. J. Millen and V. Shmatikov. Constraint Solving for Bounded-Process Cryptographic Protocol Analysis. In 8th ACM Conf. on Computer and Communications Security (CCS'01), pages 166-175, 2001.

20. F. Nielson, H. R. Nielson, and H. Seidl. Cryptographic Analysis in Cubic Time. In TOSCA 2001 - Theory of Concurrency, Higher Order Languages and Types, volume 62 of ENTCS, Nov. 2001.

21. L. C. Paulson. The Inductive Approach to Verifying Cryptographic Protocols. J. Computer Security, 6(1-2):85-128, 1998.

22. A. W. Roscoe and P. J. Broadfoot. Proving Security Protocols with Model Checkers by Data Independence Techniques. J. Computer Security, 7(2, 3):147-190, 1999.

23. M. Rusinovitch and M. Turuani. Protocol Insecurity with Finite Number of Sessions is NP-complete. In 14th IEEE Computer Security Foundations Workshop (CSFW-14), pages 174-187, June 2001.

24. C. Weidenbach. Towards an Automatic Analysis of Security Protocols in FirstOrder Logic. In 16th Internat. Conf. on Automated Deduction (CADE-16), volume 1632 of LNAI, pages 314-328. Springer, July 1999. 